\title{
Experimental Evidence for the Distinction Between Metastability and Persistence in Optical and Electronic Properties of Bulk GaAs and AlGaAs
}

\author{
M.V.B Pinheiro ${ }^{1}$ and K. Krambrock ${ }^{2}$ \\ ${ }^{1}$ Centro de Desenvolvimento da Tecnologia Nuclear (CDTN) \\ CP 941, R. Prof. Mário Werneck s/n, 30.123-970 Belo Horizonte, MG, Brazil \\ ${ }^{2}$ Departamento de Física, ICEx, Universidade Federal de Minas Gerais (UFMG) \\ CP 702, Av. Antônio Carlos 6627, 30.123-970 Belo Horizonte, MG, Brazil
}

Received February, 1999

\begin{abstract}
In this paper we present experimental evidence supporting that persistent behavior in the electronic and optical properties of bulk GaAs and AlGaAs can have different origins as: the metastability of a defect, the persistent charge transfer from a defect to another defect, and the transfer from a defect to the conduction band. In particular, we show three different cases in which defects are either directly or indirectly related to persistent changes of the optical absorption, the magnetic circular dichroism of the absorption (MCDA) and the photoconductivity (PC). The three cases are as follows: the transfer of EL2 to the metastable state EL2 ${ }^{0 *}$ in semi-insulating (SI) GaAs causing persistent quenching of the absorption and photoconductivity (PPCQ); the persistent transfer of electrons from stable EL2 ${ }^{0}$ to arsenic vacancies in lightly n-type GaAs; and the correlated appearance of persistent MCDA and photoconductivity in AlGaAs due to the photoionization of DX-centers.
\end{abstract}

\section{Introduction}

Point defects in semiconductors often cause persistent behavior in the electronic and optical properties of a semiconductor during transfer from ground to the excited state. That is the case of the well known DX centers in AlGaAs [1-3] and the EL2 defects in GaAs [4]. Both have charge states which are related to a large lattice relaxation $[5,6]$. The transfer of the DX centers by light at low temperature is accompanied by a persistent photocurrent (PPC) $[7,8]$, whereas that of EL2 is related with a persistent quenching of the photocurrent (PPCQ) [9]. In the literature, much confusion still exists when it concerns with persistent effects in the electronic and optical properties of a semiconductor. New metastabilities of defects were suggested, although only persistent electronic or optical properties were observed [10]. The main reason for that is the lack of structure-sensitive experimental data like, for example, electrically or opticallly detected magnetic resonance (EDMR and ODMR), which correlate unambiguosly the measured property with the microscopic structure of a specific defect [11]. In this work we discuss whether persistent effects like PPCQ, PPC and persistent quenching of the absorption are directly or indirectly related with the metastability of a specific defect or caused by a charge transfer process between two defects or one defect and the conduction band. For this purpose we give experimental evidence for three distinct cases: excitation of the EL2 defect in SI GaAs by light of energy between 1.0 and $1.4 \mathrm{eV}$ generating PPCQ and persistent quenching of the absorption in GaAs [12,13]; charge transfer between EL2 defects and As vacancy-related defects by light of energy below 1.0 $\mathrm{eV}$ causing also a persistent quenching of the absorption in GaAs [14]; and PPC as well as the persistent MCDA caused by photoionization of Te-related DX-centers in AlGaAs by light of energy above $0.7 \mathrm{eV}$ [8]. All those processes are observed at low temperatures. As experimental techniques, we used the transient behavior of the Magnetic Circular Dichroism of the Absorption (MCDA) correlated with Optically Detected Magnetic Resonance (ODMR), as well as optical absorption and photocurrent measurements.

\section{The case of EL2 in GaAs}

The EL2 defect is one of the most studied intrinsic defects in GaAs [4]. It appears mainly in semi-insulating GaAs grown by liquid encapsulated Czochralski (LEC) and horizontal or vertical Bridgman (HB or VB, re- 
spectively) [4]. Although its microscopic structure is still a controversial subject, it is accepted that its core is an arsenic antisite (AsGa) [15]. Acting as a double donor, it has three charge states EL2 ${ }^{\circ}, \mathrm{EL}_{2}{ }^{+}$and EL2 ${ }^{++}$which introduce two ionization levels in the band gap $\left(E_{v}+0.75 \mathrm{eV}\right.$ for $0 /+$ and $E_{v}+0.54 \mathrm{eV}$ for $+/++)$. Its neutral charge state has an excited state, EL $2^{0 *}$, which is metastable and optically and electrically inactive at low temperatures. The most accepted model for the metastable state of EL2 is that caused by a large lattice relaxation (LLR) of the As antisite in a $<111>$ direction [6]. This relaxation-induced metastability is created, at low temperature, when light of photon energy between 1.0 and $1.4 \mathrm{eV}$ transfers the $\mathrm{EL} 2^{0}$ from ground state to the excited state EL20*. It can be recovered by heating the crystals up to $140 \mathrm{~K}$. This metastability is also responsible for one of the the most well known fingerprints of the EL2 defect, the persistent quenching of photoconductivity [9]. Another fingerprint of the EL2 defect which is also related to the metastability is the persistent quenching of the MCDA band of paramagnetic EL2 ${ }^{+}[12,13]$. Figs. 1(a) and 1(b) show, respectively, the MCDA of EL2 ${ }^{+}$and the absorption of the EL2 ${ }^{0}$ in semi-insulating LEC GaAs before and after photo-quenching for illuminating light of $1.2 \mathrm{eV}$. This photo-induced quenching of the absorption and the MCDA is persistent for hours at low temperatures $(<140 \mathrm{~K})$. Figs. 2(a) and 2(b) show the transient behavior of the MCDA of EL2 ${ }^{+}$at $0.95 \mathrm{eV}$ and the transmission at $1.3 \mathrm{eV}$, which is related to the absorption band of EL2 ${ }^{0}$. Both EL2 ${ }^{0}$ and $\mathrm{EL} 2^{+}$are converted to $\mathrm{EL} 2^{0 *}$ according to the following reactions $[12,13]$ :

$$
\begin{gathered}
E L 2^{0}+h \nu \rightarrow E L 2^{+}+e^{-} \\
E L 2^{+}+h \nu \rightarrow E L 2^{0}+h^{+} \\
E L 2^{0}+h \nu \rightarrow E L 2^{0 *}
\end{gathered}
$$

The first transition is responsible for the photoconductivity (PC), and the last two for the persistent photo-quenching of the photoconductivity (PPCQ) or even a p-type persistent conductivity. While the two reactions in (1) and (2) compete with each other, the third one occurs always when the bleaching light is around $1.2 \mathrm{eV}$. As a consequence, both EL $2^{0}$ and EL2 ${ }^{+}$ are converted into the metastable state $\mathrm{EL} 2^{0 *}$ and their concentrations, which are directly related to the intensity of the MCDA and the absorption, decrese persistently $[12,13]$.
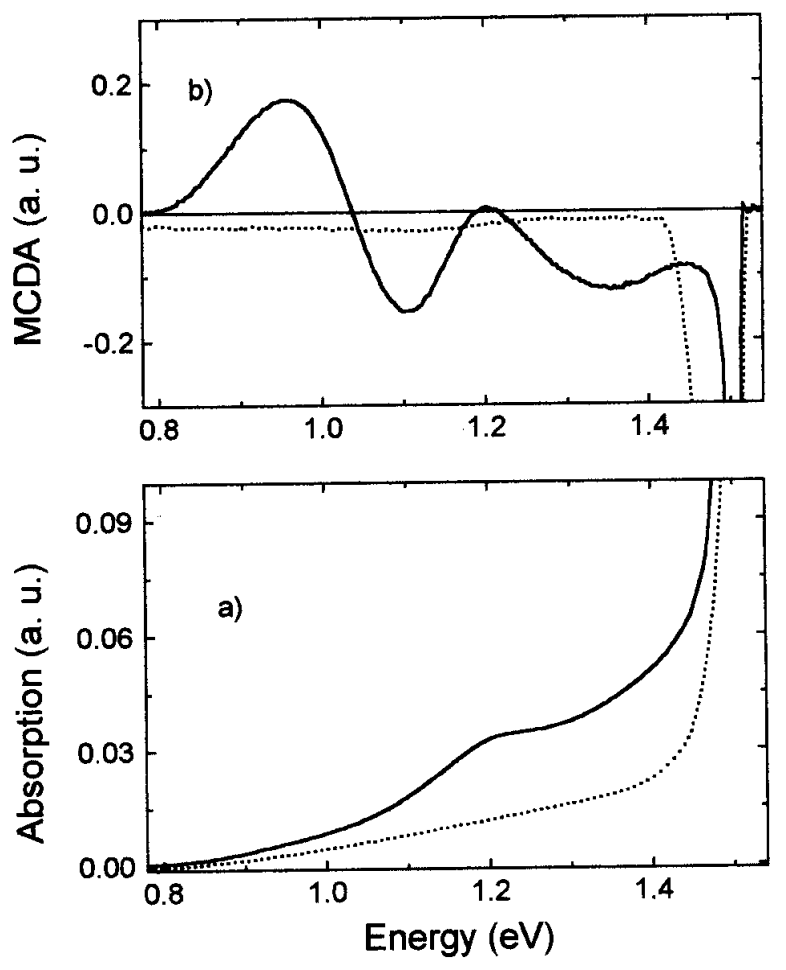

Figure 1. Optical absorption (a) and MCDA (b) in semiinsulating LEC GaAs before (solid lines) and after (dotted lines) illumination with photon energies at $1.2 \mathrm{eV}$ at low temperatures [13].
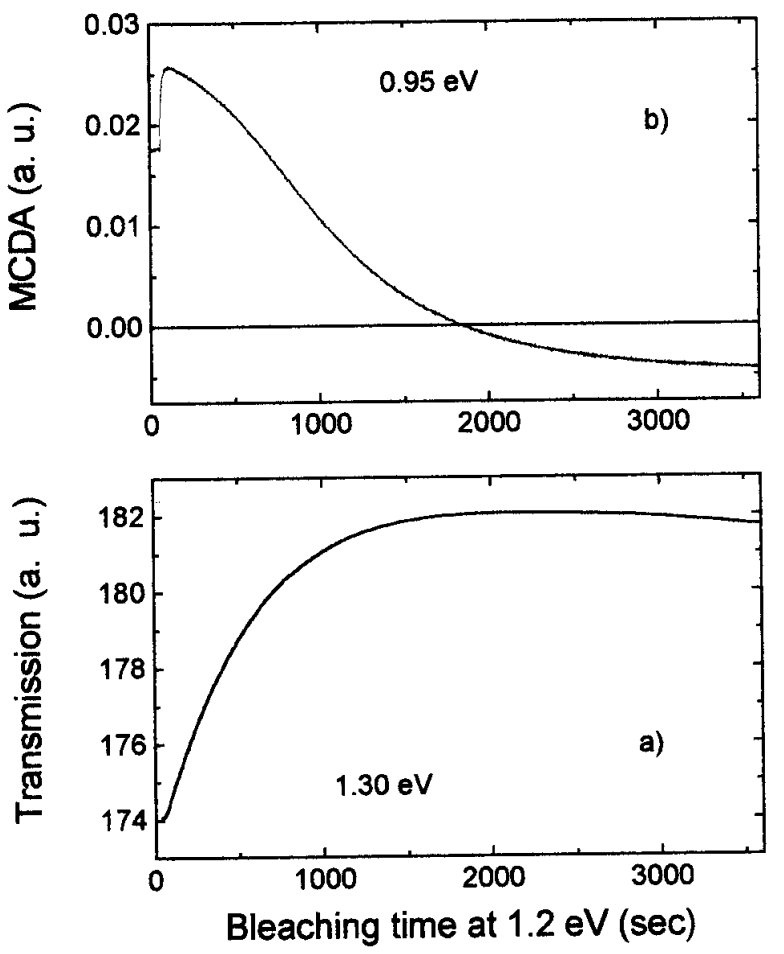

Figure 2. Transient behavior of the optical absorption at $1.3 \mathrm{eV}$ (a) and the MCDA at $0.95 \mathrm{eV}$ (b) during bleaching with light of energy of $1.2 \mathrm{eV}$ in semi-insulating GaAs at low temperatures [13]. 


\section{The charge transfer between EL2 and $V_{A s}$ in GaAs}

In a slightly n-type Te-doped GaAs sample we observed a completely different persistence which is not related to the metastability of the EL2 defect [14]. The persistence was created by light of energy below $1.0 \mathrm{eV}$ with the transfer of electrons from the $\mathrm{EL} 2^{0}$ defect to another defect, the $\mathrm{V}_{A s}$, at low temperatures (up to 120 $\mathrm{K}$ ), according to the reactions:

$$
\begin{gathered}
E L 2^{0}+h \nu \rightarrow E L 2^{+}+e^{-} \\
V_{A s}^{+}+2 e^{-} \rightarrow V_{A s}^{-}
\end{gathered}
$$

This effect has been observed in transient MCDA experiments where the MCDA band of EL2 ${ }^{+}$was persistently increased (Fig. 3) [14]. The photon energies used to occupate persistently the EL2 ${ }^{+}$states, 0.80 and $0.94 \mathrm{eV}$, were below the threshold energy for bleaching the $\mathrm{EL} 2^{0}$ centers which is above $1.0 \mathrm{eV}[12,13]$. However, one must remember that for the same sample we have also observed the bleaching of EL2 centers due to the transfer to the metastable state. This transfer was only detected for illumination with photon energies above $1.0 \mathrm{eV}$ [14]. As for the metastable transition of the EL2 centers, the persistent charge transfer from EL2 to VAs may also generate a PPCQ, which was not measured up to now. In this picture, the electrons photo-excited from the EL $2^{0}$ generate a photoconductivity which is quenched by their capture into $\mathrm{V}_{A s}$.

\section{The case of DX centers in AlGaAs}

The DX centers in n-type $\mathrm{Al}_{x} \mathrm{Ga}_{1-x} \mathrm{As}(x>0.3)$ are another kind of defect which introduce persistent properties by illumination at low temperatures in the semiconductor. Unlike the EL2 defect in GaAs, the DX center in $\mathrm{Al}_{x} \mathrm{Ga}_{1-x} \mathrm{As}$ is an extrinsic defect. Its ground state, in the most accepted picture, is a negatively charged deep donor which undergoes a large lattice relaxation (LLR) along a $<111>$ direction for group IV impurities (Sn, Si), or one of its first Ga neighbors for group VI donors (Se, Te) [7]. After photo-excitation by light of energies above $0.7 \mathrm{eV}$ at low temperatures, the deep ground state DX-becomes ionized and the released electrons are trapped in shallower substitutional donor states which may include or not the same impurityrelated effective mass type states, $d_{0}[7]$. The equilibrium between these shallow states and the conduction band generates a photoconductivity which is persistent for long periods of time when temperatures are kept below $150 \mathrm{~K}$ (PPC) [7,8]. Most group IV and group VI donor impurities behave in such a way. For Te-doped $\mathrm{Al}_{x} \mathrm{Ga}_{1-x}$ As we correlated the persistent occupation of neutral effective mass type Te-donors, responsible for a MCDA band in the near infrared region [8], with the appearance of the PPC after photoionization of DXcenters. Both the MCDA measured at $1.5 \mathrm{~K}$ and the PPC measured at $40 \mathrm{~K}$ are persistent up to $150 \mathrm{~K}$, appear with the same photon energy threshold and saturate with similar illumination times (Fig. 4). The PPC could only be detected above $40 \mathrm{~K}$ because for lower temperatures the photoexcited electrons were freezed out in the shallow $\mathrm{Te}^{0}$ states [8]. The DX-related persistence is different from the other two cases discussed above since it is not due to a metastable transformation whitin one and the same defect neither due to a charge transfer between two defects. It can be considered, in principle, as a consequence of a bistability of a defect which can be either negatively and positively charged. The reaction that describe this behavior is

$$
D X^{-}+T e^{+} \leftrightarrow T e^{0}+T e^{+}+e_{C B}
$$

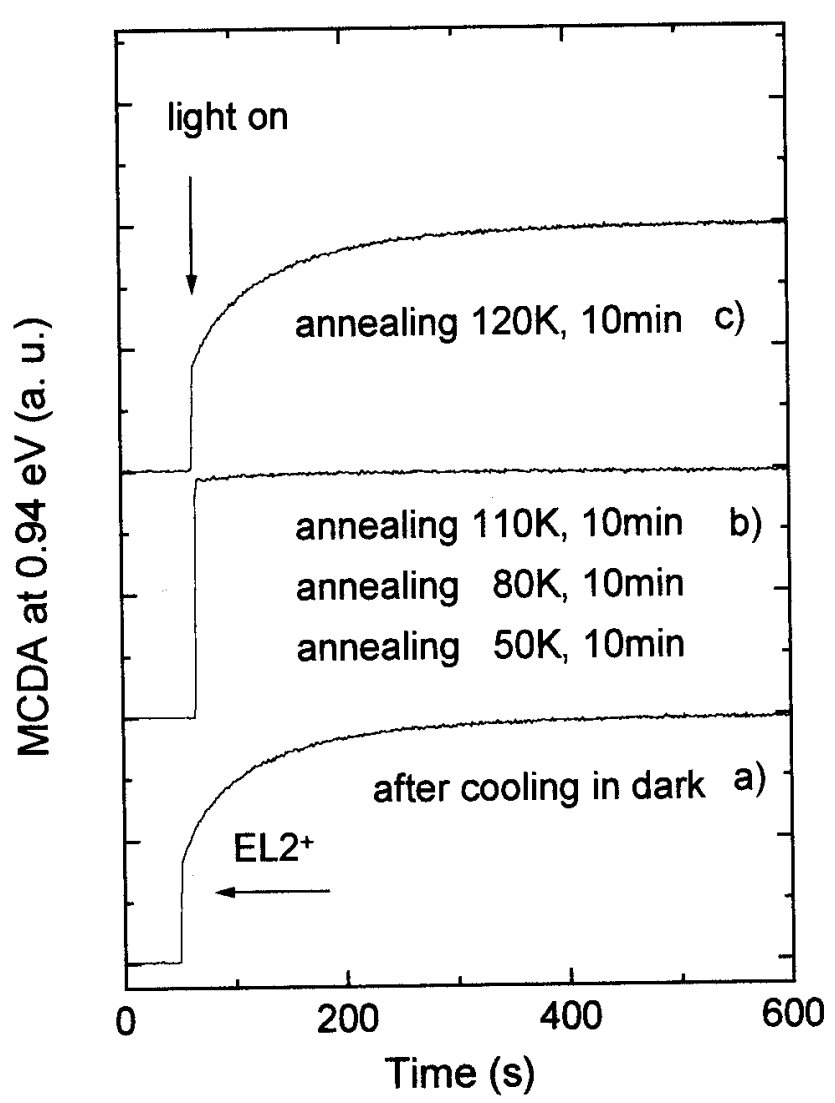

Figure 3. Transient MCDA of $\mathrm{EL}_{2}{ }^{+}$detected at $0.94 \mathrm{eV}$ during illumination with light at $0.8 \mathrm{eV}$ at low temperatures in n-type Te doped GaAs [14]. The occupation of EL2 ${ }^{+}$is persistent until $120 \mathrm{~K}$. 


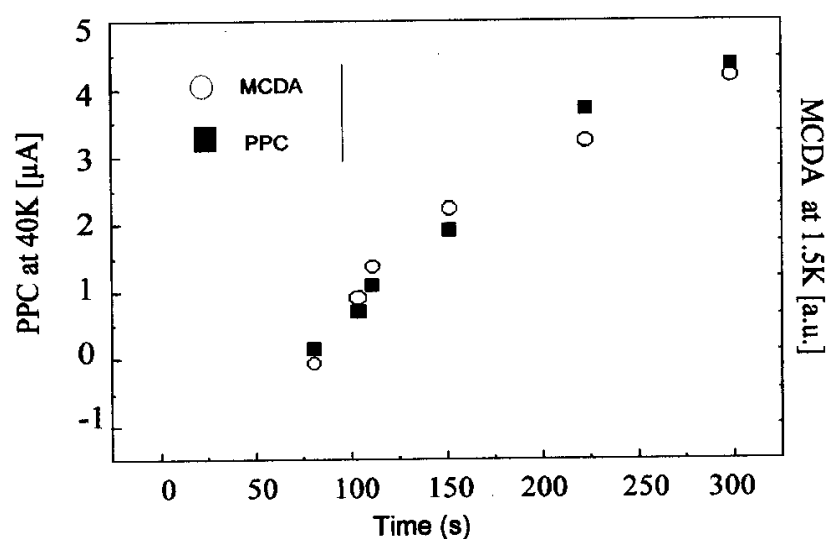

Figure 4. Correlated appearance of PPC and MCDA in Tedoped $\mathrm{Al}_{0.46} \mathrm{Ga}_{0.54}$ As indicating that after photoionization of DX centers electrons are persistently transferred to the conduction band. For $T<40 \mathrm{~K}$ these electrons are trapped in the shallow $\mathrm{Te}^{0}$ effective mass-type states [8].

\section{Discussion and conclusions}

Fig. 5 shows schematically for the three cases described above how persistent changes in the electric and optical properties are originated. The three cases are: transfer of stable EL $2^{0}$ to metastable EL2 ${ }^{0 *}$ in GaAs originating PPCQ; transfer of electrons from EL2 ${ }^{0}$ to As vacancies in slightly n-type GaAs; and PPC based on the bistability of DX centers in AlGaAs. Although there are many similar persistent effects in the optical and electrical properties of GaAs and AlGaAs, at least three different kind of phenomena originating such persistence have been identified using transient MCDA, optical absorption and photoconductivity measurements in correlation with optically detected magnetic resonance. They could unambiguously be identified because structuresensitive techniques were applied.

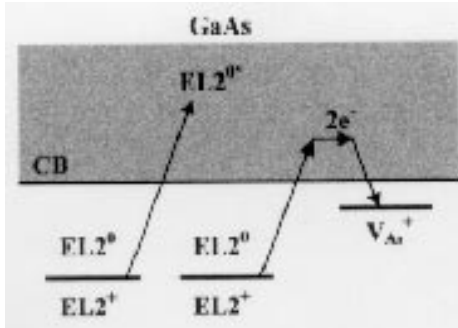

(a)

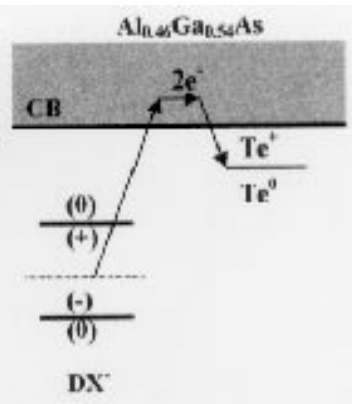

(c)
Figure 5. Schematic representation of persistent effects observed in optical and electrical properties: (a) metastability of EL2 in SI GaAs, (b) charge transfer between EL2 and As vacancies in n-type GaAs and (c) bistability of DX centers in AlGaAs.

\section{Acknowledgements}

We are grateful to Prof. J.-M. Spaeth (University of Paderborn, Germany) for many useful discussions and to FAPEMIG as well as PRONEX for financial support.

\section{References}

[1] Muñoz Merino E (1994) DX Centers-Donors in AlGaAs and Related Compounds (Defects and Diffusion Forum 108) ed Muñoz Merino E (Fug. Scitec Publications).

[2] J. C. Bourgoin, (1990) Physics of DX Centers in GaAs Alloys (Defects and Diffusion Forum ed. J. C. Bourgoin (Vaduz, Sci-Tech Publications).

[3] W. Jantasch and R. A. Stradling (1991) DX-Centers and other Metastable Defects in Semiconductors (Semicond. Sci. and Technol. 6B) ed W. Jantasch and R.A. Stradling.

[4] M. O. Manasreh and D. W. Fischer Phys. Stat. Sol. (b), 154, 11 (1989).

[5] D. J. Chadi and K. J. Chang (1988) Phys. Rev. Lett. 61, 873 (1988); D. J. Chadi and K. J. Chang Phys. Rev. B 39, 10063 (1989).

[6] D. J. Chadi and K. J. Chang (1988) Phys. Rev. Lett. 60, 2187 (1988); J. Dabrowski and M. Scheffler Phys. Rev. Lett. 60, 2183 (1988).

[7] J-M. Spaeth and M. Linde (1994) DX Centers-Donors in AlGaAs and Related Compounds (Defects and Diffusion Forum 108) ed. E. Muñoz Merino (Fug: Scitec Publications) chap. 2.

[8] M. V. B. Pinheiro, M. Linde, H. Ohkura and J-M. Spaeth Semicond. Sci. Technol. 11, 63 (1996).

[9] G. Vincent and D. Bois, Solid State Comm. 27, 431 (1978).

[10] W. C. Mitchel and J. Jiménez, J. Appl. Phys. 75, 3060 (1994).

[11] J-M. Spaeth, J. R. Niklas and R. H. Batram, (1992) Structural Analysis of Point Defects in Solids (Springer Series in Solid State Physics 43) (Berlin: Springer).

[12] M. Heße, F. K. Koschnick, K. Krambrock and J-M. Spaeth, Solid State Comm. 92, 207 (1994).

[13] K. Krambrock and J-M. Spaeth, Semicond. Sci. Technol. 13, 1100 (1998).

[14] K. Krambrock, M. V. B. Pinheiro, K-H. Wietzke and J-M. Spaeth, Mat. Sci. Forum 258, 1015 (1997).

[15] J-M. Spaeth and K. Krambrock, ) Festkörperprobleme 33, 111 (1993). 